\section{Tumors lose their privileges}

\section{By Tim Fulmer, Staff Writer}

A key challenge for cancer vaccine development is the ability of tumors to evade the immune system. Research published by a group at the University of Pennsylvania identified a key target in the process-the endothelin B receptor on tumor endothelial cells - that could provide an adjunct strategy for overcoming characteristics of the solid tumor microenvironment that lower vaccine efficacy, such as hypoxia, high interstitial fluid pressure and low extracellular $\mathrm{pH}{ }^{1}$

In the paper, published in the January issue of Nature Medicine, Ronald Buckanovich and colleagues showed that blocking the endothelin B receptor in mice with ovarian cancer resulted in an increase in tumor-infiltrating lymphocytes (TILs). Moreover, the increased homing of TILs to tumor cells resulted in the improved efficacy of a cancer vaccine. Mice treated with a cancer vaccine and a small-molecule endothelin B receptor antagonist had better antitumor responses than those receiving the vaccine alone.

The researchers used BQ-788, a generic endothelin B receptor antagonist known to have antitumor activity in animal models of breast cancer, ${ }^{2}$ melanoma, ${ }^{3}$ Kaposi's sarcoma ${ }^{4}$ and ovarian cancer. ${ }^{5}$ The vaccine consisted of UV-irradiated ID8 murine ovarian cancer cells.

The researchers suggested that endothelin B receptor overexpression "can afford immune privileged status to tumors" and that blocking the receptor "could be therapeutically useful for tumor immune therapy."

The paper builds on previous research that showed an inverse relation between TILs in ovarian cancer tumors and patient survival. ${ }^{6}$ Based on that work, the Penn group compared the gene expression profiles of endothelial cells from ovarian cancer tumors with and without TILs and discovered that the endothelin B receptor was significantly overexpressed in tumors lacking TILs.

A logical hypothesis is that antagonizing the endothelin B receptor might induce a spontaneous antitumor response that could be used to boost the efficacy of a vaccine. To test the idea, the researchers gave normal mice a prophylactic vaccine of two subcutaneous injections of UV-irradiated ID8 ovarian cancer cells followed one week later by ID8 tumor inoculation. The mice then were treated with either BQ788 or control scrambled peptide starting two weeks from the time of inoculation.

Mice receiving vaccine plus BQ-788 had significant delays in tumor growth and increased survival compared with mice receiving vaccine alone, antagonist alone or vaccine plus control peptide.

Buckanovich, who is now an assistant professor in the Division of Hematology/Oncology at the University of Michigan, told SciBX he is interested in evaluating the ability of other endothelin receptor antagonists (ERAs) to augment tumor immunotherapy. His choices of potential candidates include both selective and dual ERAs.

Buckanovich predicted that dual antagonism of the endothelin A receptor and endothelin $B$ receptor would offer further improvements over B-only antagonism. A dual antagonist would inhibit tumor growth by antagonizing the angiogenic properties of the endothelin A receptor and simultaneously enhancing $\mathrm{T}$ cell homing to tumors through antagonism of the endothelin B receptor.

One such product is Actelion Ltd.'s Tracleer bosentan, a dual endothelin A and B receptor antagonist that is marketed to treat pulmonary arterial hypertension (PAH) and dermal ulcers. Other ERAs marketed for PAH, including Thelin sitaxsentan from Encysive Pharmaceuticals Inc. and Letairis ambrisentan from Gilead Sciences Inc., only hit the A receptor and probably would not have utility in boosting cancer vaccine efficacy.

Martine Clozel, SVP and head of drug discovery, pharmacology and preclinical development at Actelion, told SciBX that "it would make sense to consider the use of our dual ERAs in cancer-in combination with vaccines or anticancer immunological approaches."

Last December, Actelion discontinued development of bosentan to treat stage IV metastatic melanoma based on preliminary efficacy data from a Phase IIa trial that evaluated the compound as a single agent in about 90 patients. Preclinical studies of bosentan in cancer are ongoing. The company would not disclose specific cancers or whether bosentan is being tested in combination with other therapeutics.

Meanwhile, Andrea Faccibene, research associate at the University of Pennsylvania's Center for Research on Ovarian Cancer Early Detection and Cure and an author on the Nature Medicine paper, said that future research should consider the efficacy of various ERAs in combination with vaccines of higher potency than those used in the paper.

Also, the timing of ERA administration needs to be worked out. The paper looked only at treatment following vaccination, not at treatment before or during vaccination, which also could boost vaccine efficacy.

Despite the potential improvements that endothelin B receptor antagonists could offer cancer vaccines, Daniel Gold, CSO and founder of Favrille Inc., told SciBX that in most cases "finding the right antigen is still the most difficult thing in designing a cancer vaccine."

He also noted that "the difficulty with adjuvants to cancer vaccines is how to get two agents approved simultaneously, the adjuvant and the vaccine-though this could change if failures mount in the space. In any case, even the possibility of dual approval would presumably require complex, multiarm trials." 


\section{TARGETS \& MECHANISMS}

But Peter Working, SVP of R\&D at Cell Genesys Inc., pointed out that although "other elements of the tumor microenvironment can certainly affect tumor vaccine efficacy, including degree of vasculature, secreted molecules and hypoxia, it would still be very useful to determine if overexpression of the endothelin B receptor is a common mechanism whereby tumors evade host immunity."

Endothelin receptors have been implicated in other cancers besides ovarian, in particular breast cancer. ${ }^{7}$ Buckanovich said he is collaborating with Nora Disis, associate professor in the Division of Oncology at the University of Washington Medical Center, to study the activity of ERAs in mouse models of breast cancer.

Although endothelin B receptor antagonists potentially could be combined with cancer vaccines, Spectrum Pharmaceuticals Inc. expects that the opposite approach — an endothelin B receptor agonist—will have utility with chemotherapy.

The company's SPI-1620 (formerly IRL 1620) is in Phase I testing as an adjunct to chemotherapy. By agonizing the endothelin $B$ receptor, the compound is expected to transiently increase tumor blood flow and thus enhance the delivery of chemotherapeutics.

Guru Reddy, senior director of preclinical research at Spectrum, said that in contrast to the vaccine adjuvant approach, the company's approach relies on the vasodilatory effect of endothelin B receptor activation. In rats with breast cancer, SPI-1620 followed by paclitaxel resulted in $25.2 \%$ tumor progression compared with $74.9 \%$ for paclitaxel alone and $78.3 \%$ for saline controls $(p<0.001) .{ }^{8}$ Animals treated with BQ-788 had vasoconstriction and no improvement in the delivery of chemotherapeutics.

\section{REFERENCES}

1. Buckanovich, R. et al. Nat. Med.; published online Jan. 6, 2008; doi:10.1038/nm1699

Contact: George Coukos, Center for Research on Ovarian Cancer Early Detection and Cure, University of Pennsylvania, Philadelphia, $\mathrm{Pa}$ e-mail: gcks@mail.med.upenn.edu

2. Grimshaw, M. et al. Mol. Cancer Ther. 1, 1273-1281 (2002)

3. Lahav, R. et al. Proc. Natl. Acad. Sci. USA 96, 11496-1150 (1999)

4. Rosano, L. et al. Am. J. Pathol. 163, 753-762 (2003)

5. Salani, D. et al. Am. J. Pathol. 157, 1537-1547 (2000)

6. Zhang, L. et al. N. Engl. J. Med. 348, 203-213 (2003)

7. Grimshaw, M. et al. Cancer Res. 64, 2461-2468 (2004)

8. Rajeshkumar, N. et al. Breast Cancer Res. Treat. 94, 237-247 (2005)

COMPANIES AND RESEARCH INSTITUTIONS MENTIONED Actelion Ltd. (SWX:ATLN), Allschwil, Switzerland Cell Genesys Inc. (NASDAQ:CEGE), South San Francisco, Calif. Encysive Pharmaceuticals Inc. (NASDAQ:ENCY), Houston, Texas Favrille Inc. (NASDAQ:FVRL), San Diego, Calif.

Gilead Sciences Inc. (NASDAQ:GILD), Foster City, Calif. Spectrum Pharmaceuticals Inc. (NASDAQ:SPPI), Irvine, Calif. University of Pennsylvania, Philadelphia, $\mathrm{Pa}$.

University of Michigan, Ann Arbor, Mich.

University of Washington Medical Center, Seattle, Wash. 